\title{
AlFREDO CHAMORRO \\ Optimisation d'une flotte aérienne par la méthode «ATEM»
}

Revue française d'automatique, d'informatique et de recherche opérationnelle. Recherche opérationnelle, tome 3, no V3 (1969), p. 67-86.

<http://www.numdam.org/item?id=RO_1969_3_3_67_0>

(C) AFCET, 1969, tous droits réservés.

L'accès aux archives de la revue « Revue française d'automatique, d'informatique et de recherche opérationnelle. Recherche opérationnelle » implique l'accord avec les conditions générales d'utilisation (http://www.numdam.org/ legal.php). Toute utilisation commerciale ou impression systématique est constitutive d'une infraction pénale. Toute copie ou impression de ce fichier doit contenir la présente mention de copyright.

\section{Numdam}

Article numérisé dans le cadre du programme

Numérisation de documents anciens mathématiques

http://www.numdam.org/ 
R.I.R.O.

(3⿻ année, V-3, 1969, p. 67-86)

\title{
OPTIMISATION D'UNE FLOTTE AERIENNE PAR LA METHODE “ ATEM "
}

\author{
par Alfredo Chamorro \\ Commandant de l'Armée de l'Air espagnole Elève du C.I.R.O. \\ Travail réalisé durant un stage \\ au Département de Recherche Opérationnelle à Air France
}

Résumé. - Dans les domaines commerciaux, industriels et militaires, on retrouve souvent le mème problème : comment affecter à des personnes ou à des unités d'un parc d'équipements, des activités diverses d'horaires imposés, en minimisant les moyens (hommes ou machines) nécessaires pour exécuter le programme d'activités.

Le modèle proposé donne une solution de ce problème par une méthode très simple et très économique en temps ordinateur, dont l'optimalité est établie à l'aide de la théorie des graphes.

\section{INTRODUCTION}

Les Compagnies aériennes sont peut-être le type d'entreprises les plus touchées par l'évolution technologique rapide.

Des avions, qui coûtent 10 millions de dollars aujourd'hui, deviendront en effet inutilisables dans une dizaine d'années par une grande compagnie, car cette dernière, engagée dans une lutte continuelle, se doit de réaliser d'importants investissements afin de conserver et d'attirer une clientèle exigeante, qui préfère l'avion le plus rapide, le plus sûr et le plus confortable.

Des décisions sur l'achat d'un certain type et d'une certaine quantité d'avions, sur l'établissement des routes et des horaires - pour citer quelques-uns des problèmes posés - représentent la prise de graves responsabilités et l'engagement de ressources considérables sur les années à venir. 


\section{PResentation dU PRobleme}

On retrouve dans les domaines les plus variés, commerciaux, économiques et militaires, des situations de structure semblable : celles qui supposent l'affectation de personnes ou d'unités d'un parc d'équipement à des activités diverses, soumises à un certain horaire, les personnes pouvant être de qualifications identique ou différente, le matériel spécialisé ou non, l'horaire rigide ou variable, les tâches exécutées seulement par une ou plusieurs catégories de personnel ou types de matériel. Mais l'optique reste toujours la même : minimiser le nombre d'éléments (hommes ou machines) utilisés dans l'exécution du programme.

A cette catégorie appartient le problème de la réalisation d'un programme de vols avec le minimum d'avions possible. Ceci étant supposé résolu, on pourra dimensionner correctement la flotte future à partir des études prévisionnelles de trafic à moyen et long terme. A court terme, cela permettra aussi de dégager le maximum d'avions disponibles en réserve ou pour l'entretien.

\section{DEFINITIONS ET CONSIDERATIONS DE BASE}

3.1. - Un vol sera l'élément unitaire et indivisible du programme, et sera défini par une suite de six ou huit données (selon que la période étudiée est une journée ou une semaine) :

$e d_{i}=$ escale de départ du vol $i$;

$h d_{i}=$ heure de départ du vol $i$;

$j d_{i}=$ jour de départ du vol $i$

$t a_{i}=$ type d'avion nécessaire pour réaliser le vol $i$;

$t e_{i}=$ type d'équipement commercial nécessaire ou souhaitable;

$j a_{i}=$ jour d'arrivée du vol $i$;

$h a_{i}=$ heure d'arrivée du vol $i$;

$e a_{i}=$ escale d'arrivée du vol $i$.

Exemple : yol 32 : «départ PARIS à 12 h 25 lundi avion Caravelle classe $1 / T$, arrivée lundi à $13 h 55$ à MADRID $\%$.

Lorsque pour des raisons commerciales ou techniques, on désire maintenir une certaine correspondance entre deux ou plusieurs vols, exemple : (PARIS $\rightarrow$ NICE $) \rightarrow($ NICE $\rightarrow$ BASTIA), cette liaison sera introduite et traitée dans le programme comme un vol simple, exemple : (PARIS-BASTIA), ce qui assurera la correspondance désirée.

3.2. Une rotation sera l'ense mble ordonné des vols affectés à un avion. Aucune limitation ne sera introduite dans le nombre de vols qui peuvent former une rotation. Les contraintes d'entretien ou de temps de service 
de l'équipage sont propres à la régulation, et n'entrent pas dans le cadre de cette étude. Exemple : rotation : (PARIS-LONDRES), (LONDRESFRANCFORT), (FRANCFORT-PARIS).

3.3. L'horizon sur lequel se développe le programme de vols sera appelé la période.

L'année se divise en deux saisons, bien différenciées du point de vue du transport aérien : l'hiver et l'été. Les Compagnies aériennes étudient donc deux principaux programmes de vols adaptés aux particularités de ces saisons. La période choisie devra être une image de la saison à étudier. La semaine est idéale, puisqu'elle permet de construire la saison $\grave{a}$ partir de sa propre répétition. Ce ne serait pas le cas pour un jour de la semaine et le mois est une période trop longue pour être traitée sur machine : en effet, une semaine d'été de la flotte moyens-courriers d'Air France comprend déjà plus de 1500 vols.

Étant donné le caractère répétitif de la période, il faut qu'à chaque escale le nombre d'arrivées soit égal au nombre de départs. Dans le cas contraire, la disposition des avions sur les aéroports serait différente au début et en fin de période, ce qui introduirait une distorsion aberrante dans la succession des périodes.

Il peut arriver que certains vols ne soient pas entièrement compris dans la période - ce qui est particulièrement vrai dans le cas des longscourriers. Pour que la période soit complète, il faudra couper les vols chevauchant la période en deux parties :

1) du départ réel à l'arrivée fictive à l'escale fictive de la fin de période ;

2) du départ fictif de l'escale fictive du début de période, à l'arrivée réelle.
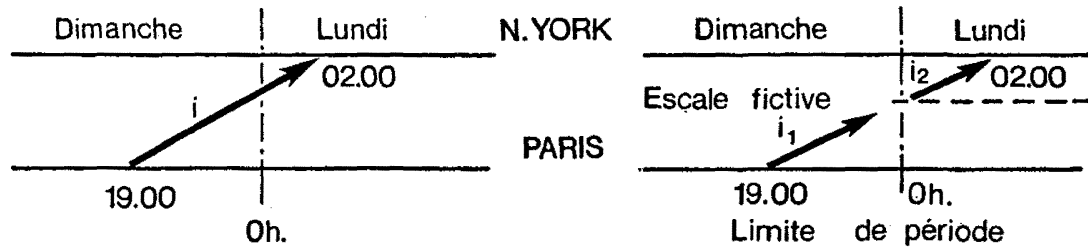

Figure 1

Le vol $i$ Paris-New York qui part à $19 \mathrm{~h} 00$ le dimanche et arrive à $02 \mathrm{~h} 00$ lundi, sera traité comme deux vols : le vol $i_{1}$ qui part de Paris à $19 \mathrm{~h} 00$ dimanche et arrive à l'escale fictive (E.F.) à $24 \mathrm{~h} 00$, et le vol $i_{2}$ qui quitte E.F. à $00 \mathrm{~h} 00$ lundi et arrive à New York à $02 \mathrm{~h} 00$ ce même jour (la période étant la semaine qui commence le lundi à $0 \mathrm{~h}$ ).

De façon exceptionnelle, la période pourra être une entité non répétitive. Exemple : la durée d'un programme de vols charters. 


\section{PROGRAMME DE VOLS A HORAIRE FIXE}

Soit le programme de vols représenté de façon schématique par la figure 2.

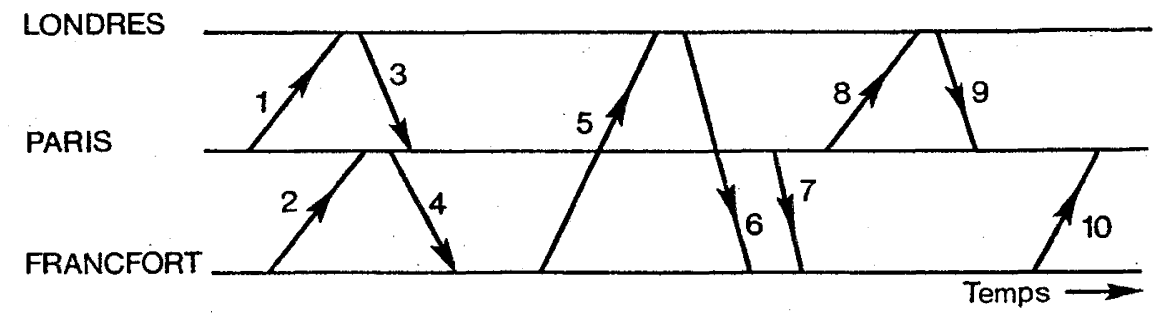

Figure 2

Chaque flêche représente un vol qui est numéroté par ordre chronologique de départ.

Chaque ligne horizontale représente une escale pendant la période étudiée.

Le temps figure en abscisse.

\section{I Graphe des liaisons possibles}

A partir du programme de vols, on définira le graphe $G=(X, U)$ représentatif de toutes les correspondances possibles. Il sera constitué :

- d'un ensemble $X$ de sommets $x_{i}$ représentant tous les vols du programme. Évidemment, $|X|=$ nombre de vols ;

- d'une application multivoque $U$ de $X$ dans $X$ représentée par un ensemble d'arcs, tels que $\left(x_{p}, x_{q}\right)$ appartient à $U$, si et seulement si le vol $x_{q}$ peut être fait après le vol $x_{p}$ par le même avion.

La nature du problème représenté par le graphe impose que le graphe soit orienté et sans circuit car le temps progresse de façon acyclique.

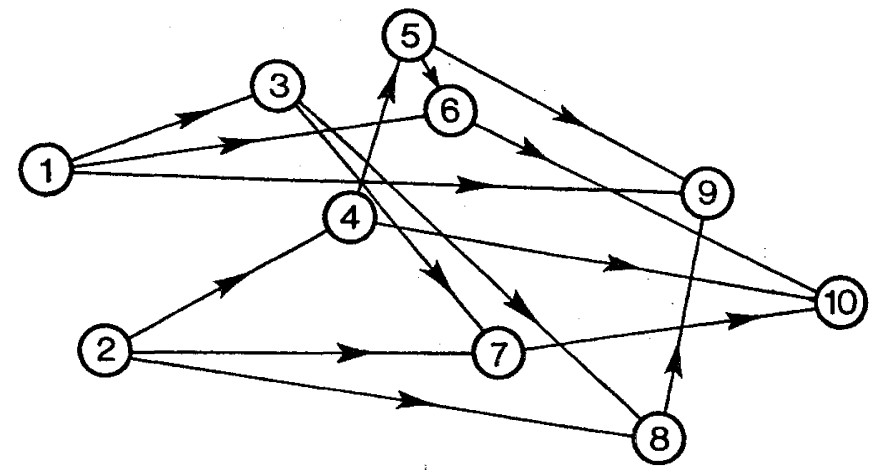

Figure 3

Graphe $G=(X, U)$, représentant toutes les correspondances possibles dans le programme de la figure 2.

Chaque sommet représente un vol.

Chaque are est une correspondance possible. 
Il faut noter que chaque chemin dans $G$ représente une rotation possible, affectable à un avion. Exemple : le chemin $\{(2,4),(4,5),(5,9)\}$ exprime une rotation composée des vols 2, 4, 5, 9. Éventuellement, un avion pourra réaliser un seul vol. Pour la rigueur de notre exposé, un sommet isolé sera aussi traité comme un chemin singulier, réduit à un seul point.

\subsection{Partition du graphe « $G$ » en chemins}

Considérons maintenant la partition (au sens des sommets) du graphe $G$ en sous-graphes $G_{i}=\left(X_{i}, U_{i}\right)$, tels que :

$-\cup X_{i}=X$

$-X_{i} \cap X_{j}=\varnothing \forall i, j$

- il existe un chemin et un seul reliant tous les sommets de $G_{i}, \forall_{i}$.

Il en résulte qu'une telle partition est aussi une décomposition du graphe $G$ en chemins disjoints, dont chacun représente la rotation assignée à un avion. En conséquence, cette partition du graphe $G$ est une certaine façon de réaliser le programme de vols.

Minimiser le nombre de tels chemins équivaut à minimiser le nombre d'auions nécessaires.

EXemple : une décomposition possible du graphe $G$ serait :

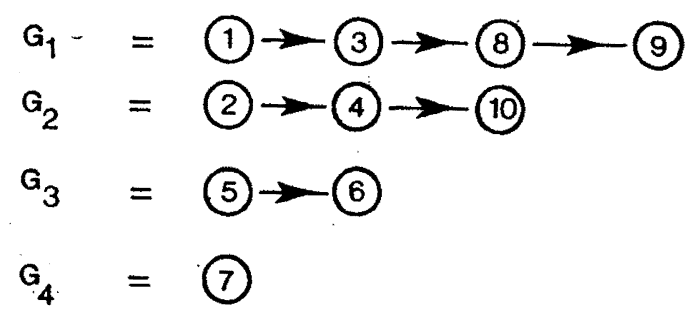

Notre but sera de trouver la décomposition d'un graphe orienté sans circuit en nombre minimum de chemins disjoints possibles (et tel que ce soit une partition au sens des sommets).

Pour cela, remarquons que :

a) Tout chemin comporte un nombre d'ares égal au nombre de sommets moins un; ceci est également vrai pour un chemin singulier (un seul sommet; aucun arc).

b) Chaque sommet appartient à un et un seul chemin. 
Si l'on désigne par

C l'ensemble des chemins disjoints,

$\mathcal{A}$ l'ensemble des arcs appartenant à tous les chemins de $t$,

$C_{i}$ le chemin $i$,

$s_{i}$ le nombre de sommets du chemin $i$,

$a_{l}$ le nombre d'arcs du chemin $i$,

on aura :

- d'après $a)$ :

$s_{i}=a_{i}+1$, et $\sum_{i=1}^{|\mathcal{E}|} s_{i}=\sum_{i=1}^{|\mathfrak{C}|}\left(a_{i}+1\right)=\sum_{i=1}^{|\mathfrak{C}|} a_{i}+|\mathfrak{C}|=|\mathcal{A}|+|\mathfrak{C}|$

- d'après $b$ ) :

$\sum_{i=1}^{|\mathcal{C}|} s_{i}=X$, avec $X=$ ensemble des vols $=$ sommets du graphe $G$, de cela résulte :

$$
|\mathcal{A}|+|\mathfrak{C}|=|X| \text {, or }|\mathfrak{C}|=|X|-|\mathcal{A}| \quad \text { (formule 4.2.) }
$$

Alors $\min |\mathfrak{C}|=\min (|X|-|\mathfrak{A}|=|X|-\max (|\mathfrak{A}|)$

Cela entraîne que minimiser le nombre de chemins revient $\dot{a}$ maximiser le nombre d'arcs appartenant à l'ensemble des chemins.

Le problème s'est donc transformé en un autre : trouver le graphe partiel $G^{\prime}=\left(X, U^{\prime}\right)$, tel que :

a) le demi-degré intérieur $d^{-}\left(x_{i}\right)$, et extérieur $d^{+}\left(x_{i}\right)$ de tout sommet soit $\leqslant 1$.

b) le cardinal de $U^{\prime}$ soit maximal.

Pour résoudre le problème, on passera au graphe biparti. En effet, rappelons que la condition exprimée en $a$ ) est caractéristique à tout couplage d'un graphe biparti et que la condition exprimée en $b$ ) l'est également pour un couplage maximum.

\subsection{Graphe biparti et couplage maximum}

Considérons maintenant le graphe $G_{b}=\left(\bar{X}, \underline{X} ; U_{b}\right)$ dans lequel

$\bar{X}=\left(\bar{x}_{1}, \bar{x}_{2}, \bar{x}_{3} \ldots \bar{x}_{N}\right)$ ensemble des vols-arrivées,

$\underline{X}=\left(\underline{x}_{1}, \underline{x}_{2}, \underline{x}_{3} \cdot . \underline{x}_{N}\right)$ ensemble des vols-départs,

$U_{b}=\left(u_{b 1}, u_{b 2}, u_{b 3}, . . u_{b M}\right)$ ensemble de toutes les liaisons possibles, sera :

Le graphe biparti correspondant au programme de vols de la figure 2, 


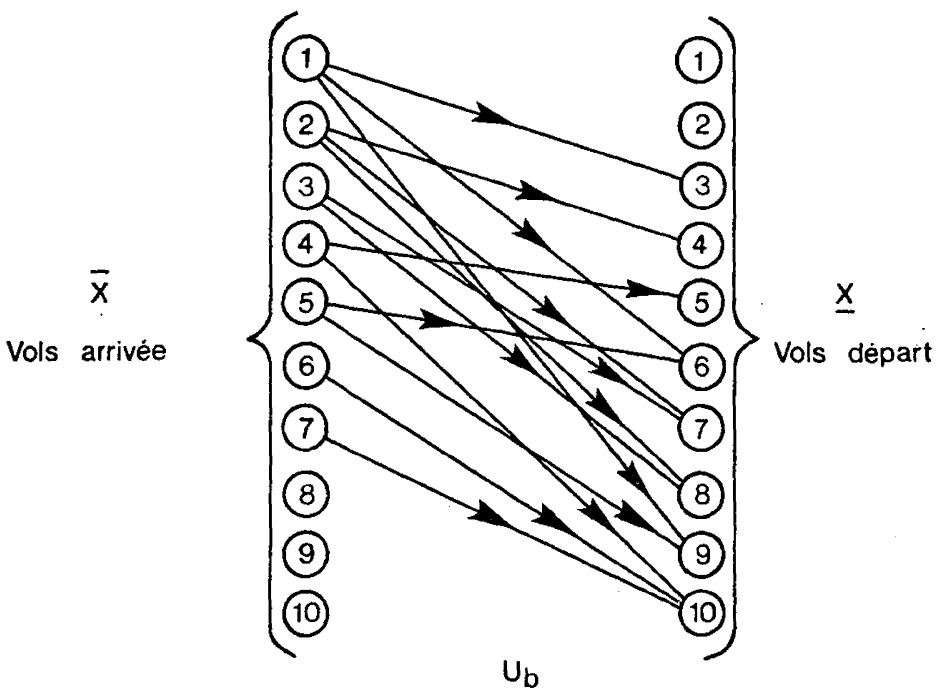

Figure 4

Graphe biparti $G_{b}=\left(\bar{X}, \underline{X} ; U_{b}\right)$, représentant toutes les liaisons possibles du programme de vols de la figure 2.

Deux vols seront liés par un arc si et seulement si ils sont susceptibles d'être effectués par le même avion.

On peut constater qu'il existe une correspondance biunivoque entre $U_{b}$ (dans $G_{b}$ ), et $U$ (dans $G$ ), et aussi que trouver l'ensemble maximum d'arcs dans $G_{b}$ tel que deux arcs quelconques ne soient pas adjacents (couplage maximum $W$ ), c'est en même temps choisir l'ensemble d'arcs $\mathcal{A}$ de cardinal maximum du sous-graphe $G^{\prime}$.

Or, le problème du couplage maximum a été déjà résolu par la méthode "hongroise " développée à partir des travaux d'Ergevary et Kuhn, et améliorée par $B$. Roy. Cette méthode sera brièvement rappelée, étant donné qu'une variante en sera employée par la suite.

\subsubsection{Procédure de couplage maximum}

Cette procédure comporte deux phases :

a) Première phase : couplage : complet"

On essaiera d'affecter chaque sommet appartenant à $\bar{X}$, à un sommet de $\underline{X}$. Lorsque aucun sommet de $\bar{X}$ ne peut plus être couplé dans $\underline{X}$, cela veut dire qu'il n'existe plus d'arc reliant deux sommets non couplés (sommets "insaturés ") et que l'on a obtenu un couplage "complet" qui sera appelé $W$.

b) Deuxième phase : couplage " maximum"

1. On formera la liste des sommets insaturés de $\bar{X}$, et celle des insaturés de $\underline{X}$ 
soit

$$
\bar{I}=\left(\bar{x}_{i 1}, \bar{x}_{i 2} \ldots\right) \text { et } \underline{I}=\left(\underline{x}_{j l}, \underline{x}_{j 2} \ldots\right)
$$

2. Si $\bar{I}=\varnothing$, alors le couplage $W$ est maximum.

Si $\bar{I} \neq \varnothing$, on prendra le premier sommet de la liste : on le marquera et on essaiera de trouver un sommet non saturé appartenant à la liste $\underline{I}$, en décrivant la chaîne alternée $\mathrm{CH}=\left\{\left(\operatorname{arc} \in\left(U_{b}-W\right)\right.\right.$, arc $\in W$ (parcouru en sens inverse), arc $\in\left(U_{b}-W\right)$, etc... $\}$.

Si l'on trouve un $\underline{x}$ non saturé, on construira un nouveau couplage :

$$
X \rightarrow[W-(\mathrm{CH} \cap W)] \cap[\mathrm{CH} \cap \bar{W}]
$$

On éliminera les nouveaux sommets saturés de leurs listes, et l'on passera à (2).

Un couplage "complet » correspondant au programme de la figure 2 pourrait être celui de la figure 5 :

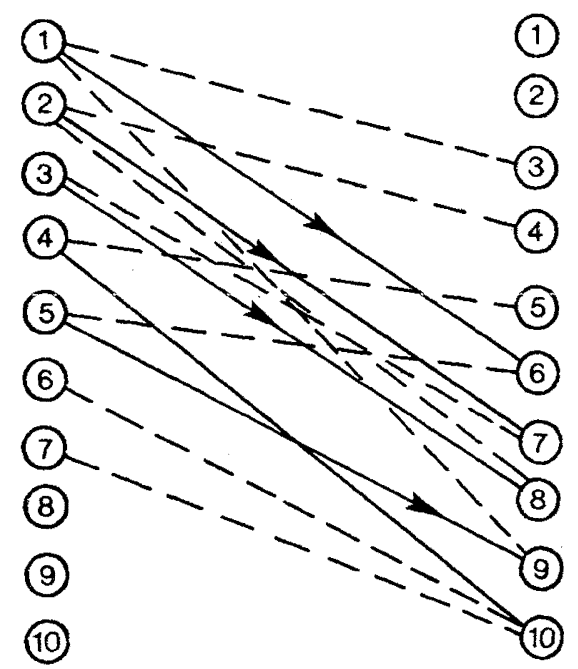

Figure 5

Couplage "complet " construit de façon arbitraire.

$W=(1,6),(2,7),(3,8),(4,10), 5,9)$.

$|W|=5$.

Le couplage " maximum " construit par l'algorithme hongrois donnerait la figure 6 : 


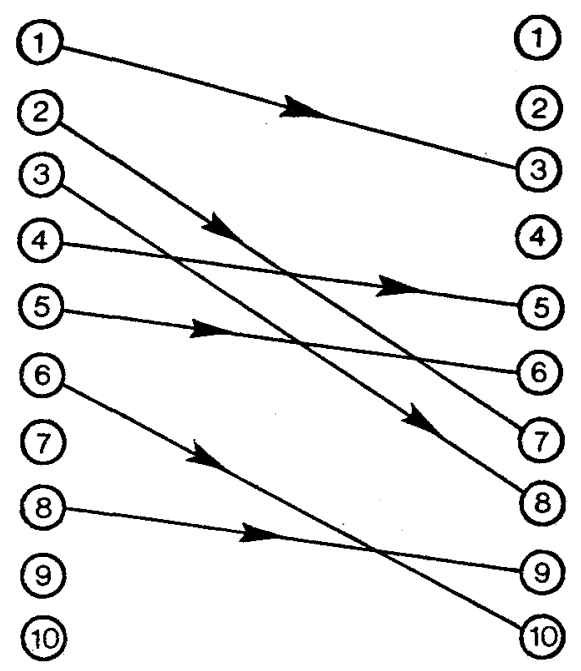

Figure 6

Couplage "maximum " réalisé à partir du couplage "complet " antérieur. $W=(1,3),(2,7),(3,8),(4,5),(5,6),(6,10),(8,9)$.

$|W|=7$.

Ce couplage représente une façon précise de réaliser le programme de vols, pour lequel on aura besoin (selon la formule 4.2) de $10-7=3$ avions. En effet :

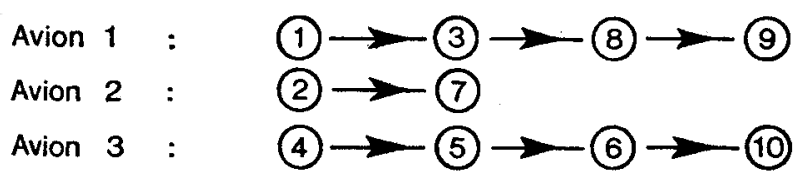

Deux remarques importantes doivent être faites :

a) Le couplage maximum n'est pas toujours unique. En général, il $\mathrm{y}$ aura plusieurs correspondances génératrices de différentes rotations ; mais le nombre des avions nécessaires sera toujours le même.

b) Le nombre de sous-graphes disjoints du graphe $G_{b}$ est borné inférieurement par le nombre d'escales qui figurent dans le programme de vols. Autrement dit, il est toujours possible de faire une partition du graphe $G_{b}$ en autant de sous-graphes disjoints $G_{b i}$ (au sens des sommets et des arcs) que d'escales figurant dans le programme de vols. 


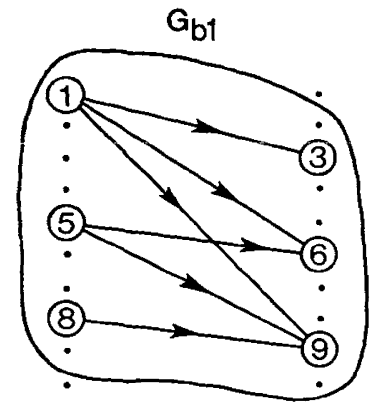

LONDRES

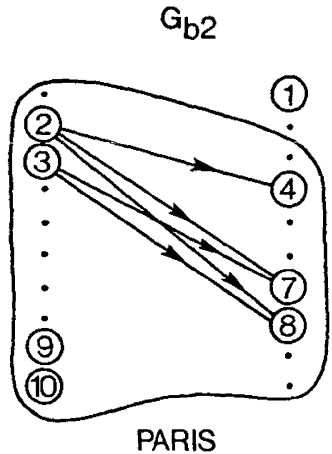

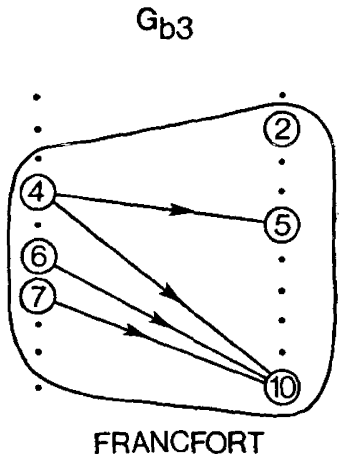

FRANCFORT

Figure 7

Partition du graphe $G_{b}$ de la figure 4 en trois sous-graphes disjoints correspondant à chacune des escales du programme de vols.

Cette propriété conduit à la conclusion suivante : le couplage maximum dans $G_{b}$ sera la somme directe des couplages maximum dans chacun des sous-graphes $G_{b i}$.

Cela sera utile pour le traitement du problème sur ordinateur. En effet, la seule matrice du graphe $G$, correspondant à une journée d'été de la flotte moyens-courriers d'Air France, occuperait 45000 positions en mémoire centrale, tandis que les matrices des sous-graphes du même programme occuperont environ 6000 positions, soit une réduction de 8 à 1 .

\subsection{Description de l'algorithme de couplage sur ordinateur}

On procédera en deux étapes sur la matrice booléenne du graphe $G$ comme nous l'avons décrit en 4.3.1.

1. Construction du couplage complet, par l'affectation de chaque ligne sur une colonne différente selon le critère fixé a priori. On marquera la case correspondant à l'affectation ligne-colonne. Si toutes les lignes ont pu être affectées, on a obtenu un couplage complet qui est aussi maximum. Sinon, on passera à l'étape suivante :

2. Construction du couplage maximum, par changement des affectations déjà établies. Ainsi, on prendra la première ligne non affectée (la 6 dans l'exemple), et on commencera - à partir d'une affectation supposée possible - un cheminement alterné sur colonne (à case marquée) et sur ligne (à case non nulle), jusqu'à arriver à une case non nulle dont la colonne ne soit pas encore affectée.

a) Si l'on y arrive, on marquera les cases de changement de direction non marquées, et l'on démarquera celles déjà marquées.

b) Si l'on n'y arrive pas, il est impossible d'affecter la ligne traitée sans perdre une affectation antérieure.

Dans les deux cas, on continue la procédure jusqu'à traiter toute la liste des lignes non affectées. 


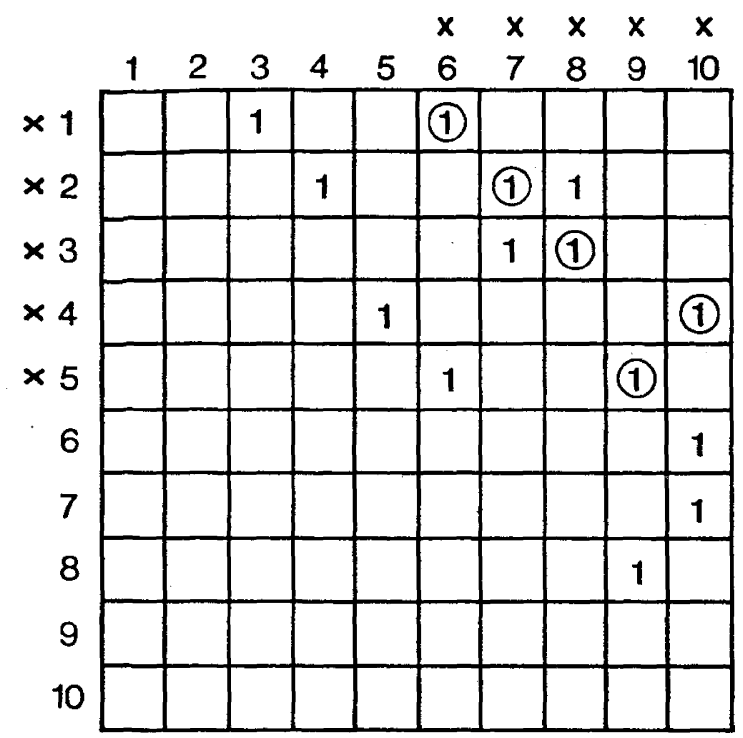

Figure $8 a$

Couplage complet de la figure 3 représenté sur la matrice booléenne du graphe $G$. On voit que les lignes non affectées, équivalentes aux sommets insaturés dans $G_{b}, \bar{I}=(6,7,8,9,10)$, ne peuvent être couplées à aucune colonne.

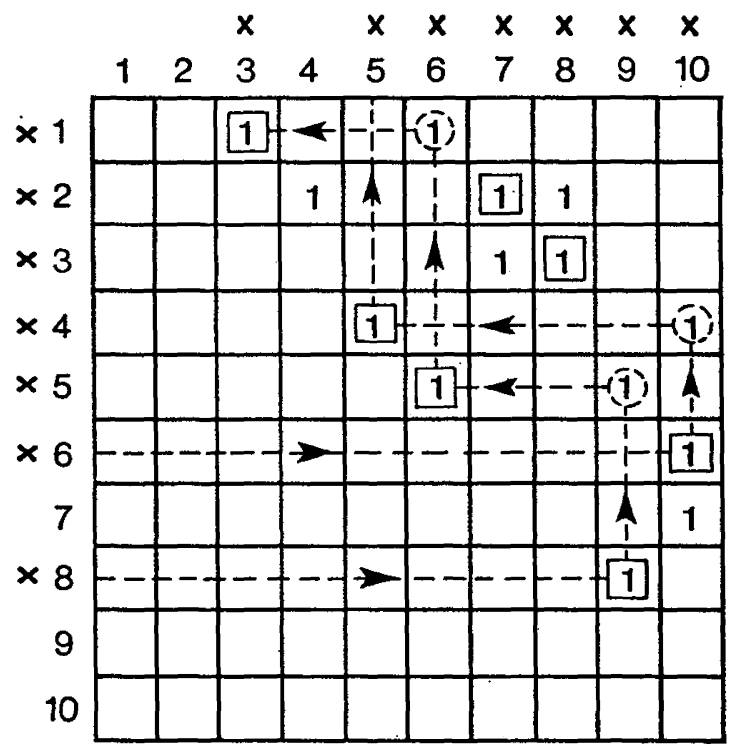

Figure $8 b$

Couplage maximum de la figure 6 représenté sur la matrice booléenne du graphe $G$.

On peut suivre les deux chaînes alternées qui ont permis d'augmenter la valeur du couplage de deux unités :

CH $1:(6,10),(4,10),(4,5)$.

CH $2:(8,9),(5,9),(5,6),(1,6),(1,3)$. 


\subsection{Inconvénients de l'algorithme}

Cet algorithme présente deux sérieux inconvénients : la rapide saturation de l'unité centrale de l'ordinateur, et le temps élevé de calcul. En effet, rappelons-nous que la période-type d'été de la flotte moyens courriers d'Air France comporte plus de 1500 vols. Mais la matrice booléenne correspondant au graphe $G$ du programme exigerait plus de deux millions d'unités de mémoire. En outre, le procédé d'exploration étant dans la deuxième étape pseudo-combinatoire, le temps de calcul augmente de façon exponentielle avec la taille de la matrice.

Le traitement du problème à partir des sous-graphes correspondant aux différentes escales (4.3), permettra dans bien des cas le passage du problème sur ordinateur, mais le temps de calcul restera encore élevé.

\subsection{La méthode « ATEM » d'acrochage à temps d'escale minimal}

Nous avons programmé cette méthode sur RAX et l'avons déjà appliquée. Elle permet d'obtenir - avec le critère d'affectation : temps d'escale minimal - un couplage complet et maximum à la fois, et cela sans s'appuyer sur la matrice booléenne du graphe $G$. On obtient ainsi l'optimisation d'emploi de la flotte, en minimisant le nombre des avions nécessaires.

La règle suivie pour établir les correspondances s'énonce ainsi :

A toute escale, chaque arrivée sera accrochée au premier départ non encore affectée.

Montrons que le couplage complet construit avec cette règle est aussi un couplage maximum. En effet, considérons pour simplifier la démonstration, une seule escale, puisqu'on a déjà vu qu'un problème multiescale est une réitération de celui d'une seule escale ; les vols sont rangés et numérotés par ordre chronologique des heures de départ.

Soit l'exemple relatif à l'escale Londres (fig. 9).

Comme les vols sont numérotés de façon chronologique, cela entraîne que si la correspondance (1) $\rightarrow$ (3) est possible, les correspondances (1) $\rightarrow$ (6) et (1) $\rightarrow$ (9) seront également possibles.

Prenons alors les vols - arripées dans n'importe quel ordre et appliquons à tous le critère " $A T E M$ ". Ainsi, par exemple, on établira les correspondances (5) $\rightarrow$ (6) (8) $\rightarrow$ (9) et (1) $\rightarrow$ (3).

Supposons qu'il reste encore un ou plusieurs sommets arrivées insaturés : soit $\bar{x}_{\ell}$ un de ceux-ci. Puisque le couplage est complet il n'existera aucun arc allant de $\bar{x}_{i}$ à un sommet départ insaturé. Soit $\underline{x}_{k}$ un des sommets terminaux des arcs qui partent de $\bar{x}_{i}$.

Pour que l'on puisse augmenter d'une unité la valeur du couplage, il faudra trouver une chaîne alternée reliant deux sommets insaturés. 
Comme $\underline{x}_{k}$ est saturé, il existera un $\bar{x}_{r}$ extrémité initiale de l'arc qui sature $\bar{x}_{k}$. Mais il n'existe aucun arc allant de $\bar{x}_{r}$ vers un sommet insaturé de $\underline{X}$. En effet, l'ensemble d'arcs partant de $\bar{x}_{r}$ vers des sommets autres que $x_{k}$ sont de deux types :

1) ceux qui vont vers des sommets $\underline{x}_{k-p}$ antérieurs à $\underline{x}_{k}$ finiront en des sommets saturés : sinon, on aurait dû établir la correspondance avec ce sommet-départ, au lieu de $\bar{x}_{k}$, par la procédure ATEM ;

2) ceux qui vont vers des sommets postérieurs à $\underline{x}_{k}$ finiront aussi en des sommets saturés. Le contraire entraînerait l'existence d'un sommetdépart $\underline{x}_{k+h}$ (postérieur à $\underline{x}_{k}$ ) non affecté, ce qui est en contradiction avec l'hypothèse du couplage complet. En effet, comme $\bar{x}_{i}$ est liè à $\underline{x}_{k}$, il le sera aussi à $\underline{x}_{k+h}$; or, l'existence d'un arc reliant les deux sommets insaturés $\bar{x}_{i}$ et $\underline{x}_{k+h}$; aurait permis d'augmenter le couplage d'une unité, et il ne serait pas - comme supposé - complet.

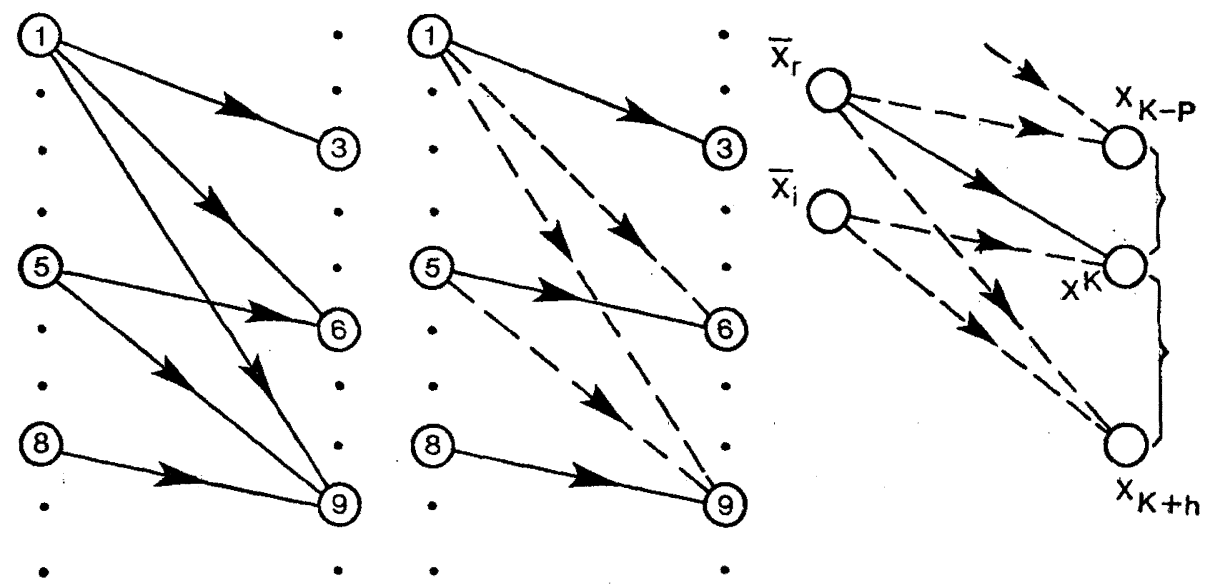

Figure $9(a, b, c)$

Situation contraire à l'hypothèse d'accrochage "ATEM ".

S'il existe $\left(\bar{x}_{i}, \underline{x}_{k}\right)$ et $\left(\bar{x}_{r}, \underline{x}_{k+h}\right)$ cela entraîne l'existence de $\left(\bar{x}_{i}, \underline{x}_{k+h}\right)$ contraire à l'hypothèse de couplage complet.

a) Graphe $G_{b 1}$.

b) Couplage complet "ATEM ".

c) Démonstration.

Nous ne pouvons donc pas trouver une chaîne alternée reliant deux sommets saturés. Il n'est donc pas possible d'augmenter d'une unité le couplage. En conséquence, s'il n'existe pas de contraintes limitatives des accrochages autres qu'un temps minimal pour le service de l'avion; la méthode ATEM donne toujours l'optimum. 


\subsection{Avantages de la méthode « ATEM »}

Par comparaison avec la méthode générale exposée en 4.4. ATEM présente deux avantages remarquables :

a) D'abord, l'occupation de mémoire centrale est minimale puisque l'algorithme s'appuie seulement sur la liste des vols, et non pas sur la matrice booléenne du graphe des liaisons possibles. On diminue donc cette occupation dans le rapport (nombre de vols) $\times$ (nombre de vols)/(nombre de vols). La raison en est que la méthode utilise un vecteur et non pas une matrice.

b) Deuxièmement, le temps de calcul est diminué de façon considérable, parce qu'on élimine l'aspect pseudo-combinatoire de l'algorithme (4.4.2) qui devient ici séquentiel (voir organigramme ATEM).

L'application de "ATEM " sur le programme de vols d'un jour d'été de la flotte moyens-courriers d'Air France, comprenant 212 vols, a demandé 3 secondes de calcul.

\subsection{Limites de la méthode}

La méthode ATEM permet de minimiser le temps perdu par une flotte d'avions identiques réalisant des vols homogènes. Si les avions sont de plusieurs types inégalement adaptés aux vols, et si les accrochages de vols en escale présentent des coûts qui ne sont pas proportionnels au temps, la méthode ATEM n'est plus adaptée. Par contre la méthode de couplage avec l'algorithme hongrois $(\$ 2)$ est optimale, en remplaçant les 1 des couplages par les coûts réels correspondants.

\subsection{Organigramme de la méthode « ATEM »}

L'application de cet algorithme sur le programme de vols de la figure 2 donnerait les rotations suivantes :

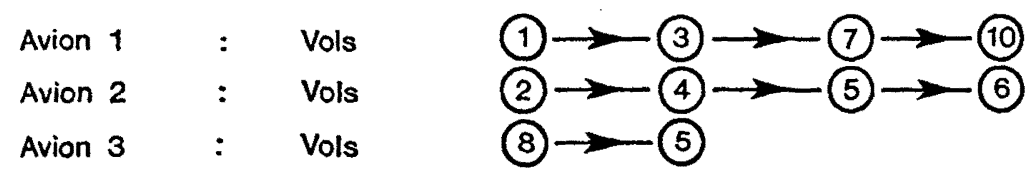

Figure 10

\section{PROGRAMME DE VOLS A HORAIRE VARIABLE}

Le problème pourrait être présenté de la façon suivante :

Étant donné un programme de vols, serait-il possible de diminuer le nombre d'avions nécessaires pour l'exécuter, par l'introduction de légères modifications dans l'horaire de certains vols?

Évidemment, il ne s'agit pas d'étudier ici toutes les possibilités de changement qu'offre un programme; d'abord, parce qu'un tel programme 


\subsection{Organigramme de la méthode « ATEM 》}

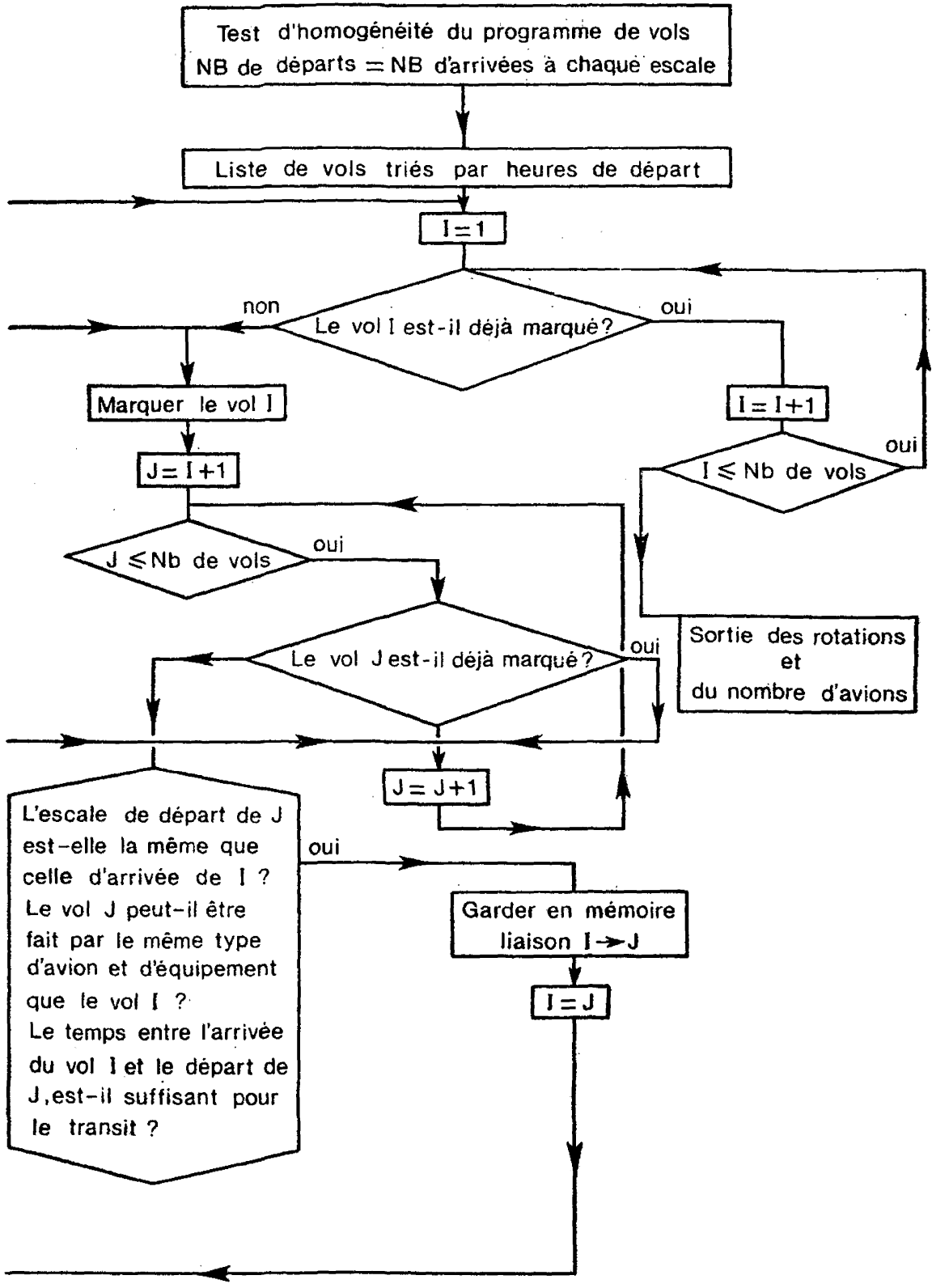


est le résultat des travaux coordonnés de plusieurs Divisions et de l'expérience acquise pendant les années passées par la Compagnie; ensuite, parce qu'en dehors d'un certain intervalle, chaque changement entraîne un nombre élevé - presque combinatoire - de mutations dans les correspondances possibles; enfin, parce que traiter ensemble tous les changements possibles - seul moyen d'arriver à l'optimum - exigerait une infinité d'itérations, le temps étant une grandeur continue.

\subsection{Méthode « ATEM 》 à horaire variable}

Cette méthode s'exécute en deux phases :

a) Un premier ajustement de l'horaire qui - en un certain sens pourrait être appelé Amélioration de l'horaire, et $b$ ) une seconde qui sera l'application directe de "ATEM" sur le programme modifié.

\subsubsection{Amélioration de l'horaire}

Prenons comme exemple le programme composé des quatre premiers vols de la figure 2. Il est évident que pour le réaliser, on aura besoin au moins de deux avions. Un avion pourra réaliser les vols (1) $\rightarrow$ (3) et un autre les vols (2) $\rightarrow$ (4) (fig. 11).
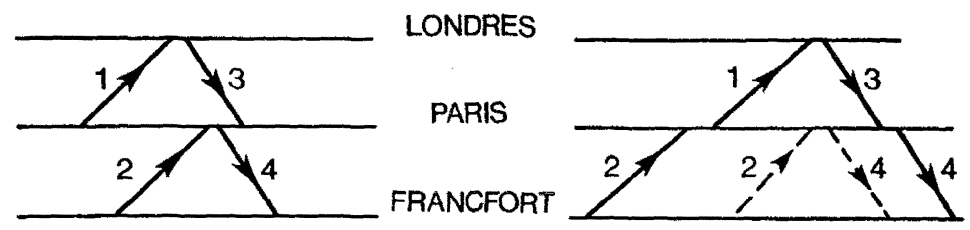

Figure $11(a, b)$

a) Programme initial de vols. Deux avions sont nécessaires.

b) Programme "amélioré ". Un seul avion suffira.

On remarquera qu'une légère avance dans l'horaire du vol 2 , et le retard du 4, permettrait la réalisation du programme par un seul avion.

Ceci est le but de la méthode : déplacer chaque vol jusqu'à la position qui optimisera le nombre de liaisons possibles, sans détruire aucune des liaisons initiales.

Pour cela, on procédera à l'étude - par ordre chronologique et séparément - de chaque vol et de son environnement, ce qui permettra de trouver et de limiter les intervalles maximaux d'indifférence à droite et $\grave{a}$ gauche, au dehors desquels tout déplacement serait accompagné d'une diminution du nombre de liaisons possibles, et l'on fixera l'horaire "amélioré " du vol dans la position la plus proche de l'horaire initial dans l'intersalle d'indifférence qui maximise les gains des liaisons (fig. 12). 


\section{LONDRES}

PARIS

FRANCFORT

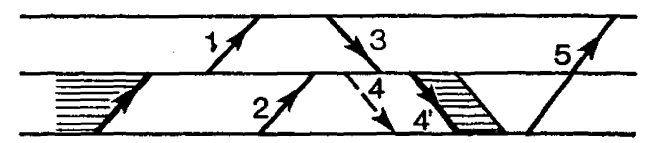

Figure 12

Traitement du vol 4 pour améliorer l'horaire. Les intervalles d'indifférence seront les zones rayées. Un déplacement du vol au dehors de la zone droite détruirait au moins une correspondance : la (3) $\rightarrow(4)$ ou la $(4) \rightarrow(5)$. Dans cette nouvelle position 4 , on a gagné la liaison $(3) \rightarrow(4)$ impossible dans l'horaire initial.

\subsubsection{Algorithme pour l'amélioration de l'horaire}

Notons d'abord que tout retard dans l'horaire d'un vol $i$ - les autres étant stationnaires - augmente ou maintient le nombre des "correspondances précédentes" possibles (vols " $h$ " qui peuvent être suivis du vol " $i$ ) et réalisés par le même avion), mais ce retard peut - en même temps - diminuer le nombre de "correspondances suivantes" possibles (vols " $j$ " qui pourraient suivre le vol " $i$ ) dans la rotation assignée à un avion).

$\mathrm{Au}$ contraire, l'avancement de l'horaire d'un vol diminuera - en général - les "correspondances précédentes" et augmentera les "correspondances suivantes ».

L'algorithme réalise le traitement séquentiel de tous les vols, qui devront passer par les cinq étapes suivantes.

Le vol sera :

1) Retardé jusqu'à : a) la limite permise par la première correspondance suivante possible, ou si celle-ci n'existe pas, jusqu'à : b) la fin de période. Ce déplacement aura créé, éventuellement, de nouvelles correspondances précédentes. La dernière de celles-ci aura établi la borne gauche de l'intervalle droite d'indifférence.

2) Avancé jusqu'à : a) la limite permise par la première correspondance précédente possible, ou si celle-ci n'existe pas, jusqu'à $b$ ) le début de période. Il est possible que de nouvelles liaisons suivantes soient établies. La première de celles-ci aura défini la borne droite de l'intervalle gauche d'indifférence.

3) Placé dans la position la plus proche de l'horaire initial appartenant à l'intervalle (gauche ou droite) d'indifférence qui maximise les gains des correspondances.

4) Reclassé dans la liste chronologique des vols.

5) Marqué, pour contrôler la séquence du traitement.

Une fois que tous les vols ont été traités, on passera à la deuxième phase de la méthode : l'application de "ATEM " au programme "amélioré » (fig. 13). 


\subsection{Organigramme de la méthode « ATEM » à horaire variable}

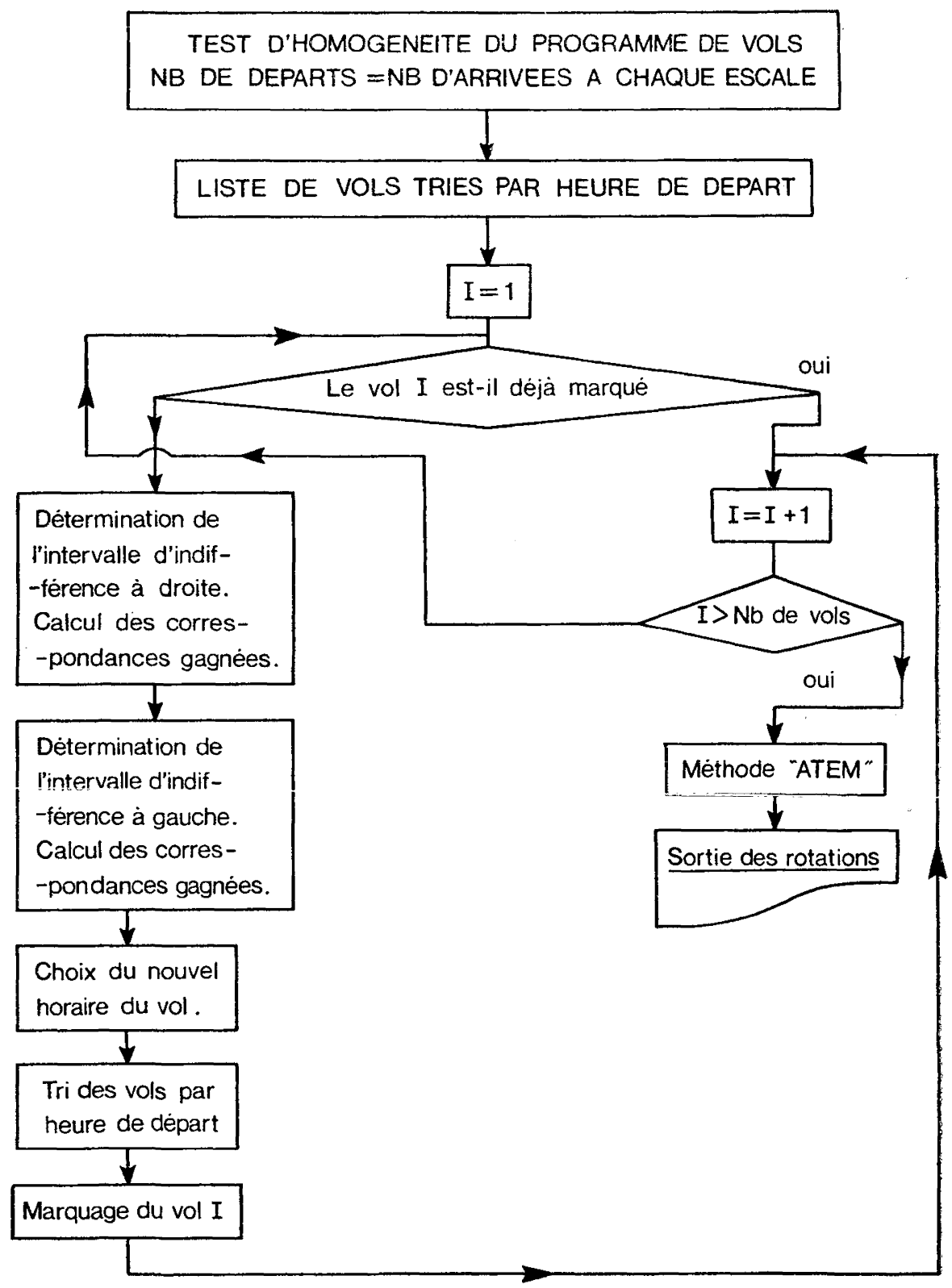

Figure 13

Organigramme de "ATEM » à horaire variable. 


\section{APPLICATION A UN CAS CONCRET}

Supposons que l'on souhaite tester un programme prévisionnel de trafic; par exemple, celui des vols longs-courriers d'Air France pour l'été de 1974.

A cette époque, de nouveaux avions comme le Boeing 747 et le Concorde seront déjà en exploitation régulière sur les lignes de la Compagnie. Or, il faut dès aujourd'hui tester les fréquences et les horaires pour rentabiliser l'emploi de tels avions.

Le premier souci sera de choisir la période représentative du programme. Or, on a vu en (3.3) l'intérêt que la semaine présente dans l'étude d'un programme saisonnier par son caractère répétitif. Mais il faut, bien sûr, normaliser la semaine pour éviter des vols qui en sortent ou y rentrent. par la méthode explicitée en (3.3).

On fera attention aux liaisons qui présentent un intérêt spécial du point de vue commercial ou technique qui seront traitées comme un seul vol (3.1).

Une fois constituée, la liste des vols qui composeront le programme sera codifiée (3.1), et ces derniers triés par ordre chronologique des heures de départ.

On définira la valeur des différents paramètres qui serviront à réguler la réalisation des accrochages : temps de transit, estimés comme les minimums nécessaires pour permettre un service correct de l'avion à l'escale. En général, il y a deux types d'escales : celles qui ont une forte densité de trafic et qui - en conséquence - exigent un plus grand temps de transit (Paris-New York, etc...) et les escales dont le trafic permet un service de l'avion assez rapide. Mais on pourra fixer un temps de transit particulier pour chaque escale.

La liste des vols et les paramètres de régulation seront les "entrées" ou données de base du programme "ATEM » sur ordinateur.

Dans la première phase "ATEM A HORAIRE FIXE ", on obtiendra comme sortie :

a) l'ensemble des rotations,

b) le nombre minimum d'avions pour exécuter le programme.

Dans la deuxième phase, chaque vol sera traité pour "améliorer " l'horaire. La nouvelle liste des vols sera maintenant l'entrée pour l'application de "ATEM ", ce qui donnera :

a) un nouvel ensemble de rotations,

b) un nouveau nombre minimum d'avions.

L'étude de ces deux ensembles de rotations, des nombres d'avions nécessaires dans les deux cas, de la valeur commerciale et technique des rotations et des modifications introduites dans l'horaire, permettront d'avoir une mesure de la qualité du programme prévisionnel étudié. 


\section{APPLICATIONS DE LA METHODE ATEM A D'AUTRES ETUDES DEJA REALISEES}

Dans deux cas autres que l'optimisation d'un programme de vols, la méthode ATEM a été utilisée :

a) Premièrement, pour l'étude prévisionnelle du dimensionnement de l'aéroport de Paris en 1980 et 1985. A partir de certaines hypothèses du trafic existant à cette date, le problème était de trouver le groupement des opérations aériennes par faisceau ou par type d'avion - ou par combinaison des deux - de façon à minimiser le nombre de postes de stationnement nécessaires, ou à les utiliser de façon régulière tout au long de la journée.

La procédure ATEM d'affecter toute arrivée au premier départ possible, compte tenu de retards traités de façon non aléatoire mais paramétrisable, a permis de tester un large nombre d'hypothèses.

b) Deuxièmement, pour la simulation des différentes hypothèses retenues à partir de l'étude précédente. Des courbes de retards aux arrivées et aux départs construites à partir des statistiques à Orly, et extrapolées sur 1980-1985 ont permis de mieux prendre en compte l'effet des conditions réelles d'opération de la future aérogare à Roissy-en-France. 